\title{
Data Object Modeling for Depreciation System Using UML
}

Asmaa Yaseen Hammo

asmahammo@uomosul.edu.iq

College of Computer Sciences and

Mathematics, University of Mosul, Iraq

Received on: 17/02/2003

\author{
Alaa F. Saeed \\ General Directorate of North \\ Electricity Distribution \\ Ministry of Electricity, Iraq
}

Accepted on: 07/10/2003

\begin{abstract}
Data object modeling is considered the most effective style in data manipulation. This style provides the ability to decompose complex systems into parts, these parts can be manipulated simply, understanding, accuracy with easy updating reducing run time errors supporting ability of design languages such as Unified Modeling Language UML. The research implements modeling of Depreciation system using UML and its tools such as (objects, classes, link \& association \& aggregation, generalization, drive data, class diagram, packages, logical horizon) and obtaining the general schema for classes to be converted to an used application using data base programming language.
\end{abstract}

Keywords: UML, Depreciation, Object, aggregation, generalization, logical horizon.

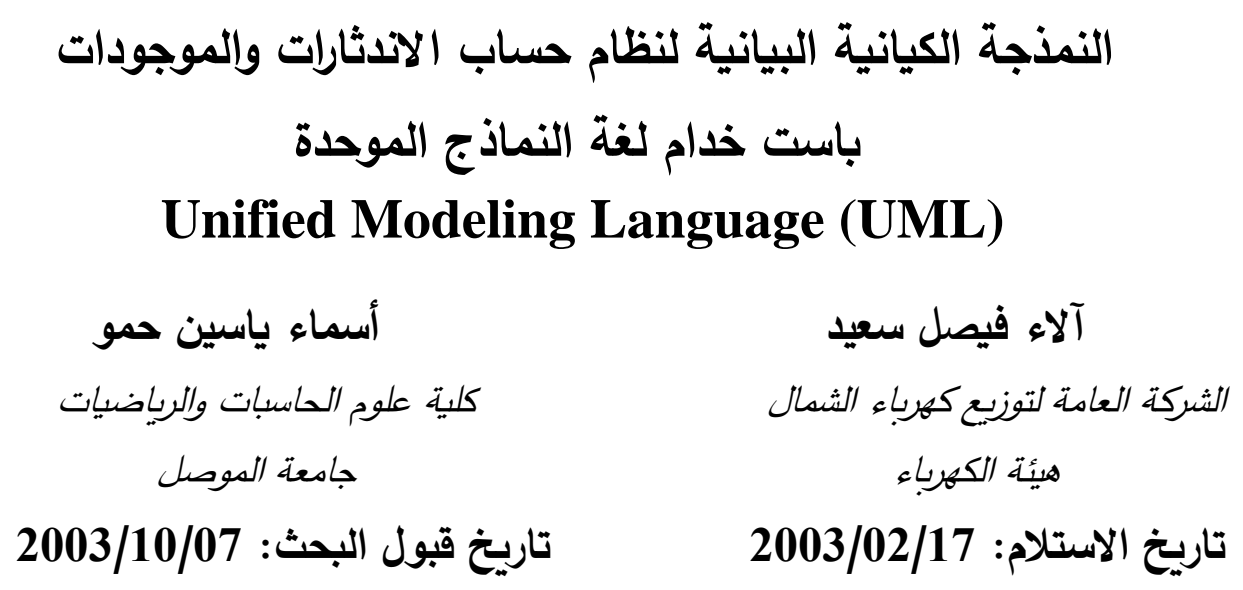

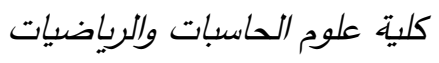

جامعة الموصل

تاريخ قبول البحث: 2003/10/07

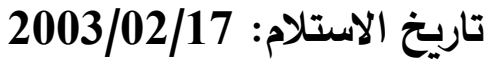




\section{الملخص}

يّعد أسلوب النمذجة البيانية من اكثر الأساليب فاعلية في التعامل مع قواعد

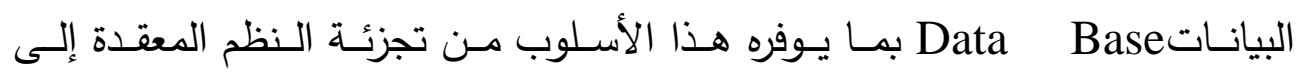
أقسام يمكن التعامل معها بيسر وفهم ودقة فضـلاً عن سهولة التعديل وتقليل أخطاء

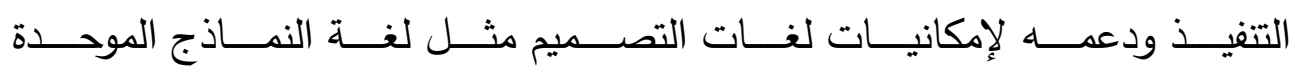

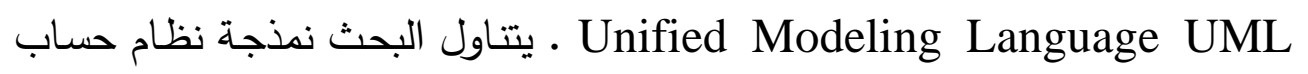
الاندثارات باستخدام لغة النماذج الموحدة و أدواتها (الكيانات ، الأصناف ، الروابط لـان

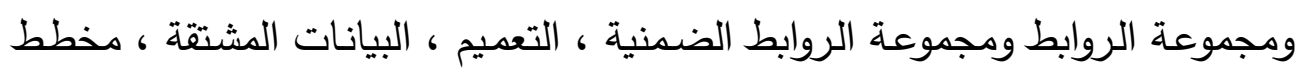

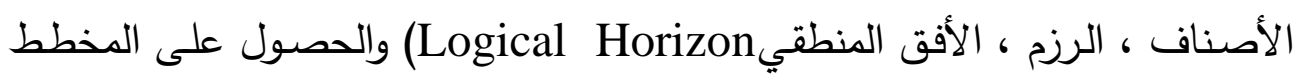

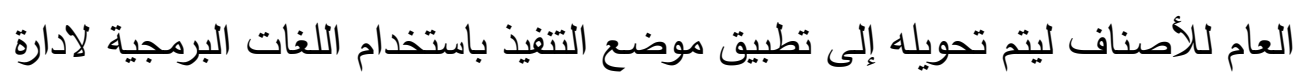
قواعد البيانات.

الكلمـات الرئيسـية: UML ، الاندثار ، الكـائن، الروابط الضــنية، التعـيم، الأفق المقدمة: المنطقي.

إن زيادة تطور المؤسسات وحجم بياناتها مع تحديث هذه البيانات واسترجاعها

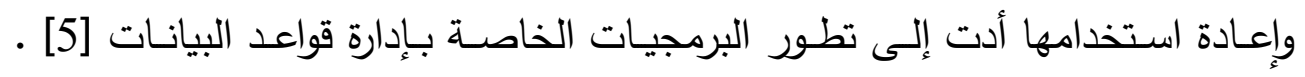

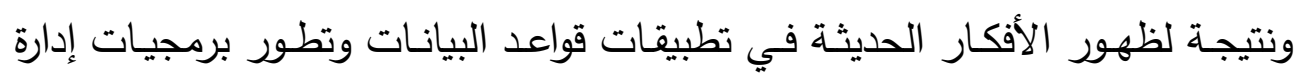
الأعمال كل ذلك تطلَب عمل نموذج من الكيانات مع إمكانية عمل تغيير عليه دون

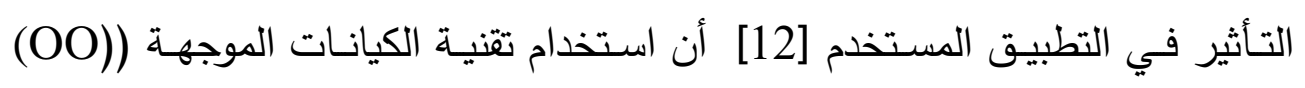
(Object Oriented

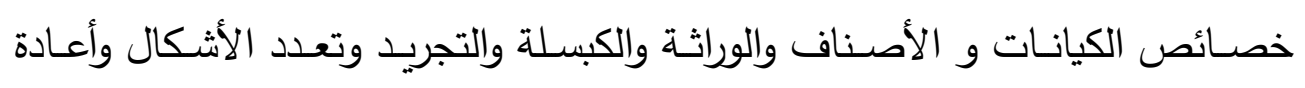
الاستخدام [17][18]. 


\section{Object Modeling}

1-النمذجـة الكيانية

النمـوذج MODEL هو ملخص لدراسـة المسألة مـن مختلف الجوانب إذ

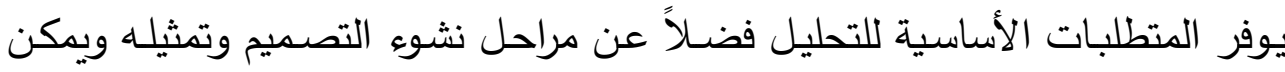
التعبير عن نموذج البيانات من خلال أنواع متعددة من المخططات الرسومية [17] ـ

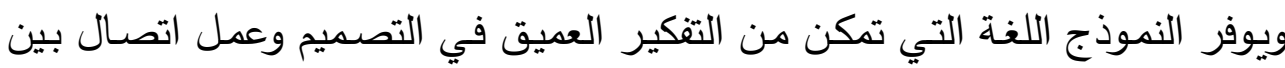
المطور والمستخدم من خلال فصل الأفكار عن تفاصيل تمثيل التطبيق • إن تصميم نمـوذج واضـح وصـريح وخاضـع للفحص الدقيق ينتج عنـه برمجيـات تكون متينـة وصحيحة كما إن لغة النمذجة المستخدمة تسمح بتعبيرات دقيقة ومحكمة للأفكار التي

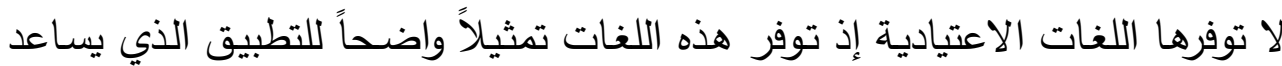
على الصيانة وإعادة الاستخدام من مكتبة البرمجيات والذي يؤدي بدوره إلى تقليل حجم البرنامج وتقليل الوقت اللازم للبرمجة [6] [9] . إن وصف العمليات التي يستخدها المطور والمحلل في تطوير التطبيق بناء Methodology Of على متطلبات المستفيد يطلق عليها منهجية علم قواعد البيانات Database بالبيانات [3] ويمثل النموذج الخاص بقواعد البيانات ويقوم بتعريف نظام إدارة قواعد البيانات Data Base Management System الذي يستخدم لتمثيل البيانات والعلاقات التي تربطها والعمليات المطبقة عليها ـ إن الغرض من استخدام نموذج البيانات هو عمل نموذج دقيق أو رسم تخطيطي يلبي منطلبات المستفيد ، ويعّد

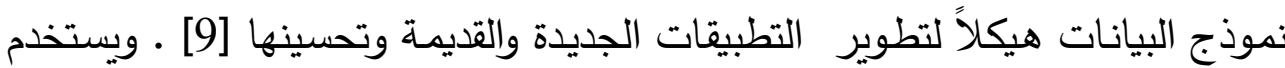
نموذج البيانات بوصفه مرحلة تمهيدية بدلاً من الدخول إلى برمجة تطبيقات قواعد

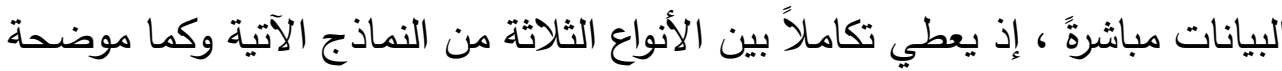

في الثكل (1)

- مــوذج المفـاهيم Conceptual Model : الذي يمثل مفـاهيم إدارة الأعمال ووجهة نظر المستخدم في التطبيق. 
- النمـوذج المنطقي Logical Model : الذي يمثل تصـيم قواعد البيانـات

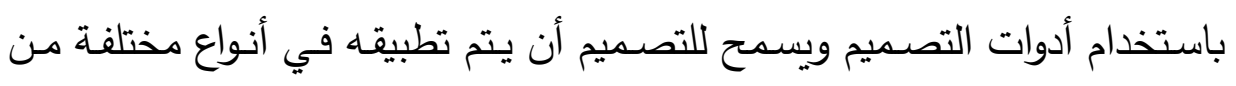

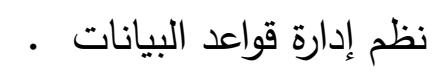

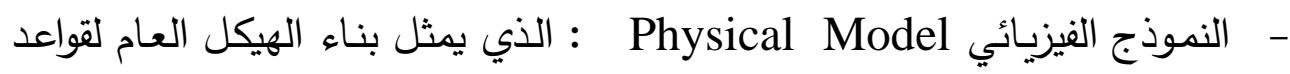

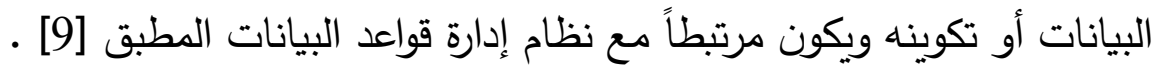

Conceptual نوذج المفاهيم

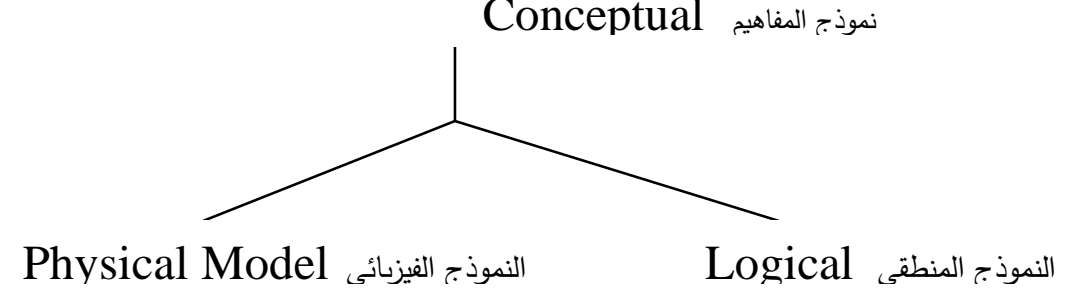

\section{الثكل (1) نماذج قواعد البيانات}

إن نظام حساب الاندثارات يسيطر على جميع ممتلكات الثركات والدوائر

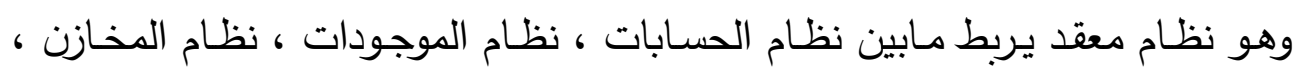

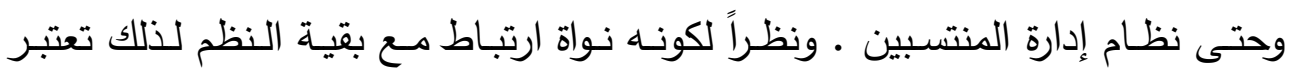

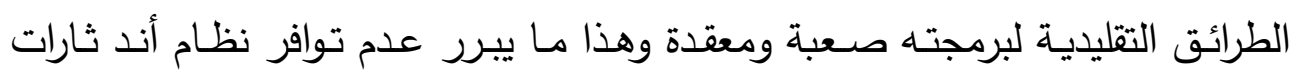

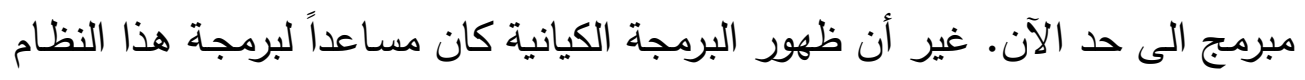

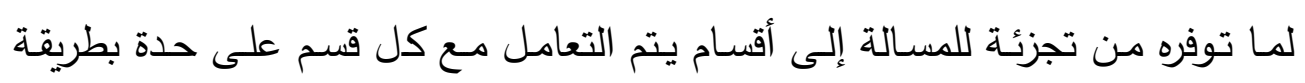
منظمة.

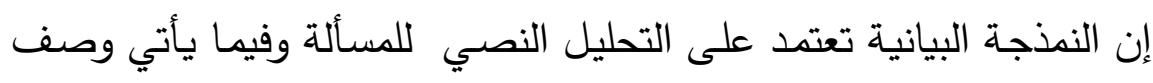

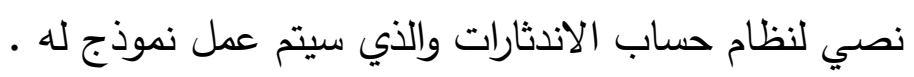
الاندثار (الاستهلاك) هو النقص التدريجي في قيمة الموجود الثابت لات سباب كثيرة

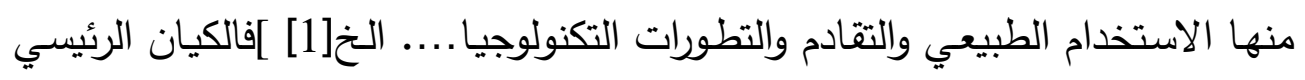

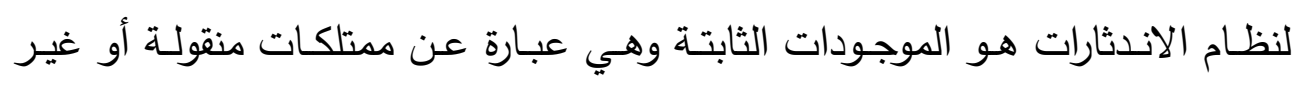

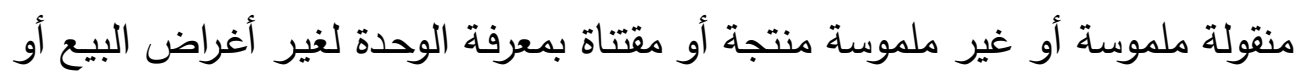

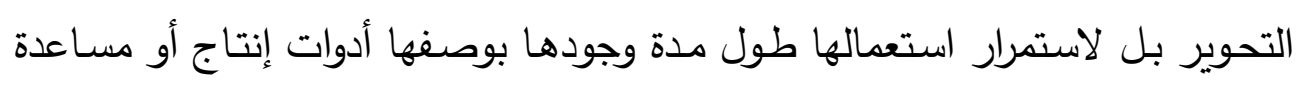


في الإنتاج.ولتعريف هذه الموجـودات في النظـام يكـون مـن خـلال صـنف حركـة

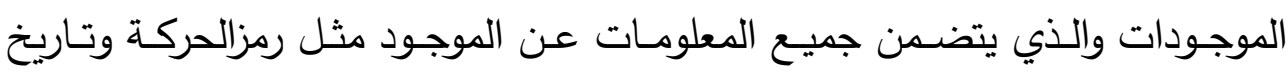

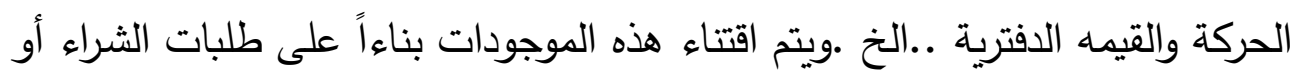

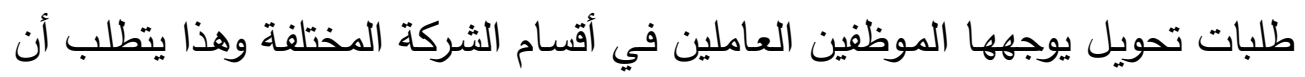

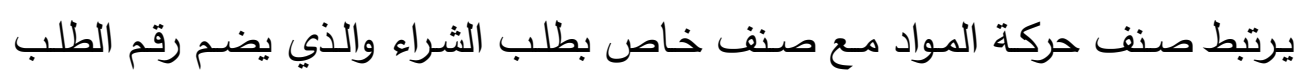

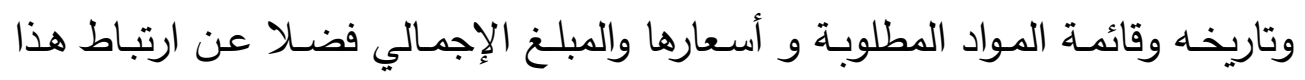
الصنف مع صنف الموظفين لتحديد معلومات الموظف مثل الاسم والعنوان الوظيفي

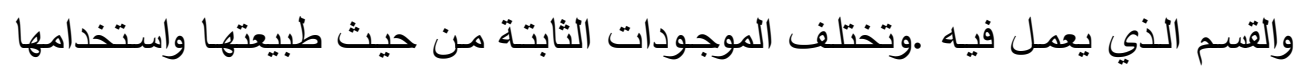

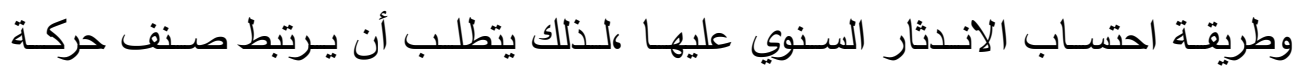

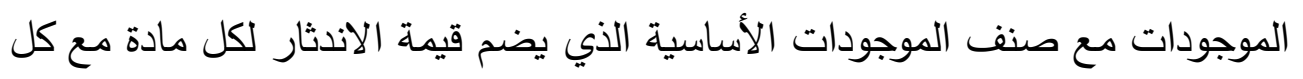
المعلومات عنها مثل الاسم والنوع والرمز ولكل الموجودات الثابتة الملموسة (المادية)

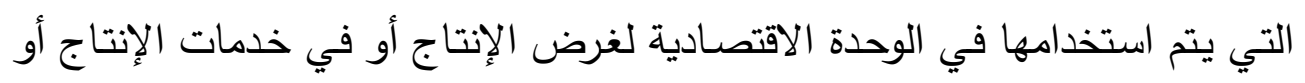

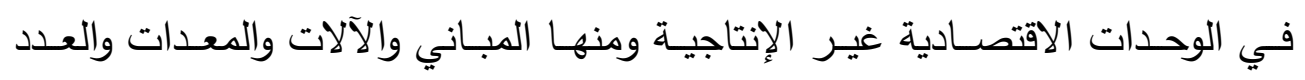

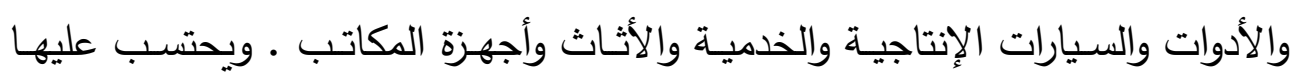

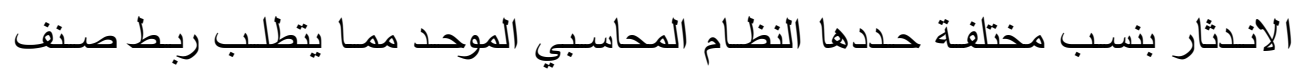
الموجودات الأساسية مع صنف خاص بنظام المحاسبي الموحد للحصول على أرقام

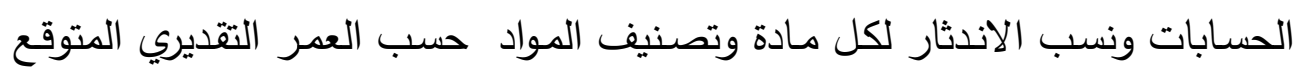
لكل من هذه الموجودات. والموجودات غير الملموسة (غير مادية) ويدخل ضمنها العلامات التجارية وبراءات الاختراع وبما حدده النظام المحاسبي الموحد.

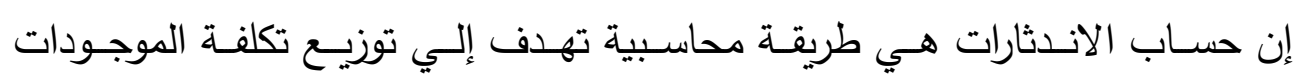

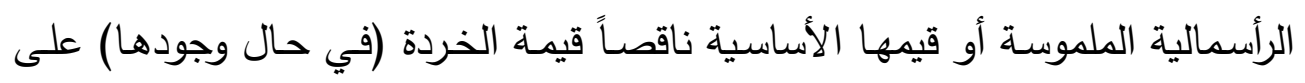

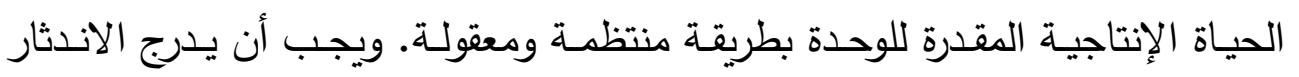

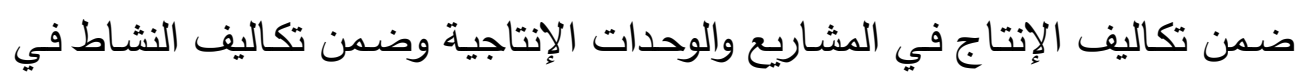
الوحدات الاقتصادية الأخرى ، حتى تحصل الوحدة الاقتصادية في نهاية المدة المالية 
على صافي الأرباح الحقيقية وذلك لان ما يفقده الموجود من قيمته إنما هو عنصر

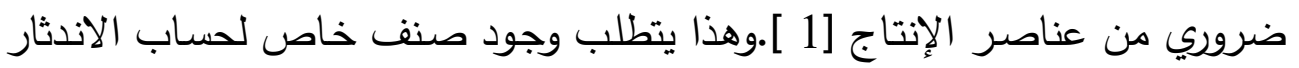

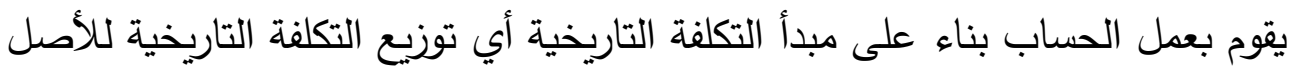
الثابت على عدد سنوات العمر الإنتاجي الدقدرة للموجود دون النظر إلى التغييرات

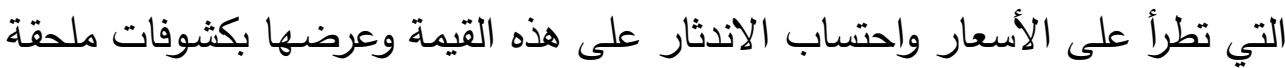

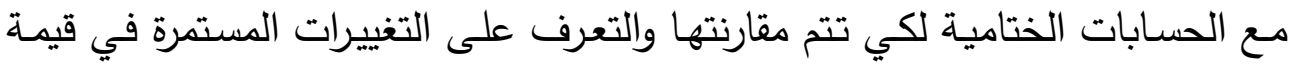

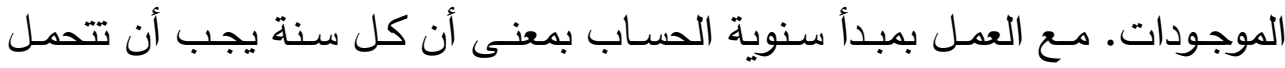

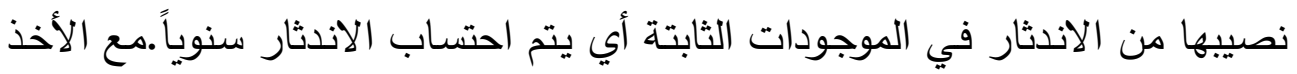

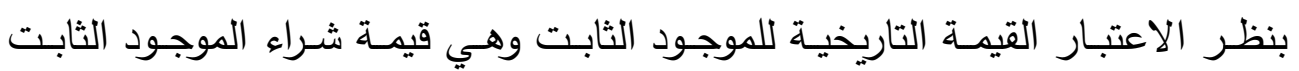

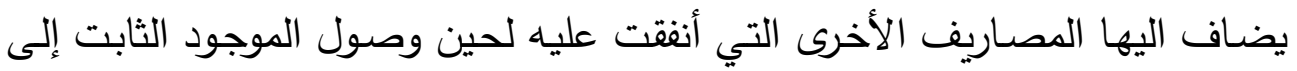

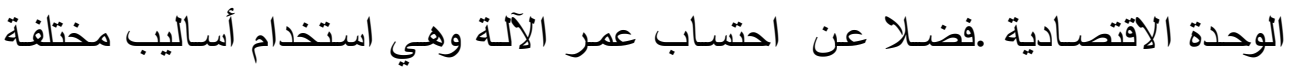
لاحتساب عمر الآلمة منها عدد ساعات التشـغيل الفعلية التي يحتسب على على أساسها

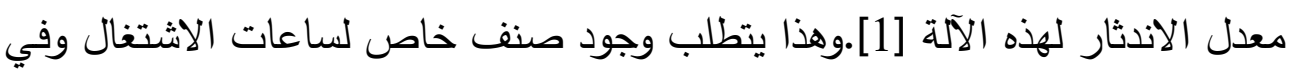
حالة انقضاء العمر الانتاجي للموجود او الالة من خلال أتلافها أو فقدانها من الثركة

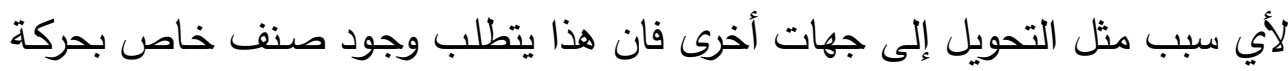
الثطب والتحويل للموجود وتتم هذه الحركة بإشراف لجان خاصة تقوم بالعملية وهذا يتطلب الارتباط مع صنف اللجان وما يتضمنه من معلومات عن تاريخ تثكيل اللجنة

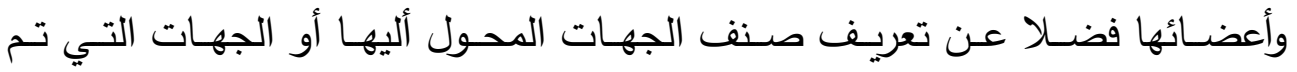
الاستلام منهـا ـ ولكون هذه الموجودات يستخدمها الموظفون كـالأدوات المكتبيـة

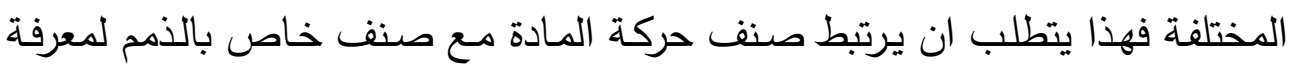
الموظف المستخدم لهذا الموجود والقسم العامل فيه وهذا يتطلب عمل ربط بين هذه الاصناف باستخدام علاقات ذات تعدديات مختلفة. 


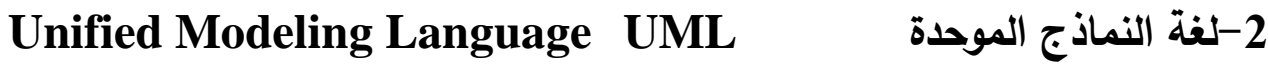

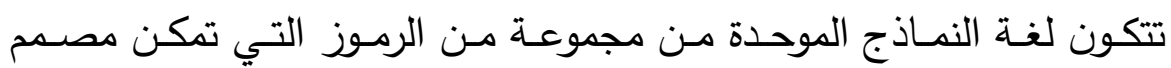

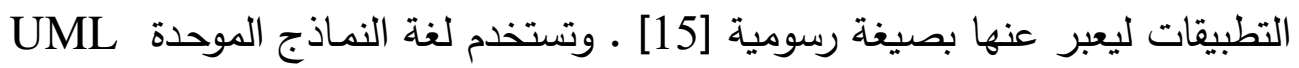

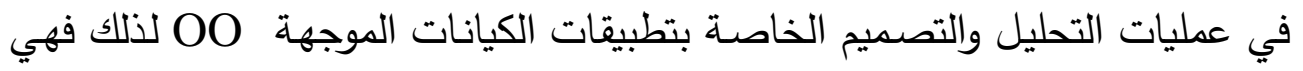
تقدم ميكانيكية جديدة في الحصول على نموذج الأصناف Class Model الذي يعّد

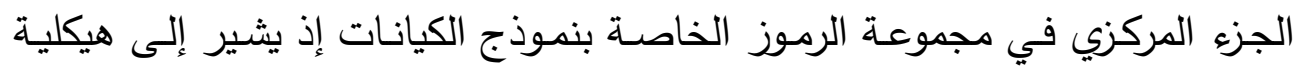

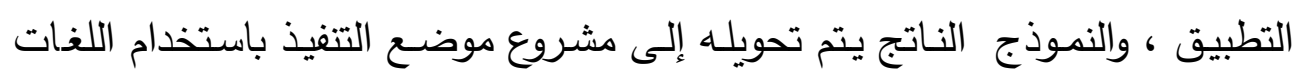

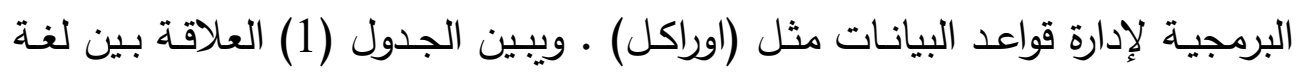

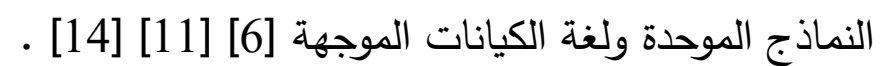

\begin{tabular}{|l|l||}
\hline \multicolumn{1}{|c|}{ OO } & \multicolumn{1}{c|}{ UML } \\
\hline Class & Object Type or Abstract Data Type (ADT) \\
\hline Attribute & Object Type Attribute \\
\hline Operation & Object Type Method \\
\hline
\end{tabular}

الجدول (1) العلاقة بين لغة النماذج الموحدة ولغة الكيانات الموجهة

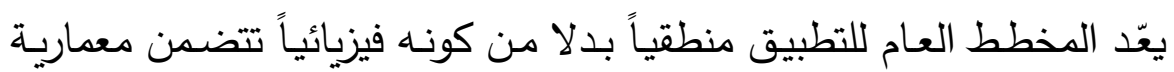

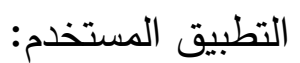

طبقة النمـاذج Model Layer : تتكون من النموذج الخاص بالنظام مثل

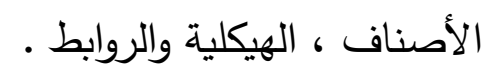

طبقة الدوال Functional Layer : تحتوي على الدوال التي تطبق على النظام للتحول من طبقة النماذج إلى طبقة الاتصال .

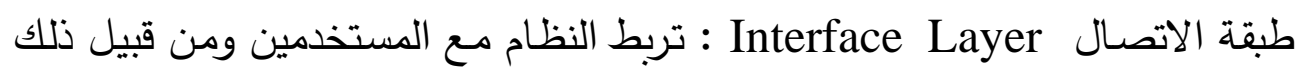
استخدام التقارير والواجهات [21] ـ

ويوضـح الثكل (2) عملية معالجة الصيخ المكونـة للتطبيق والمكتوبـة بلغـة لتوليد شفرة البرنامج التي تخزن في طبقة النماذج الخاصة بالتطبيق كالهيكل

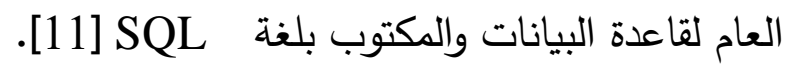




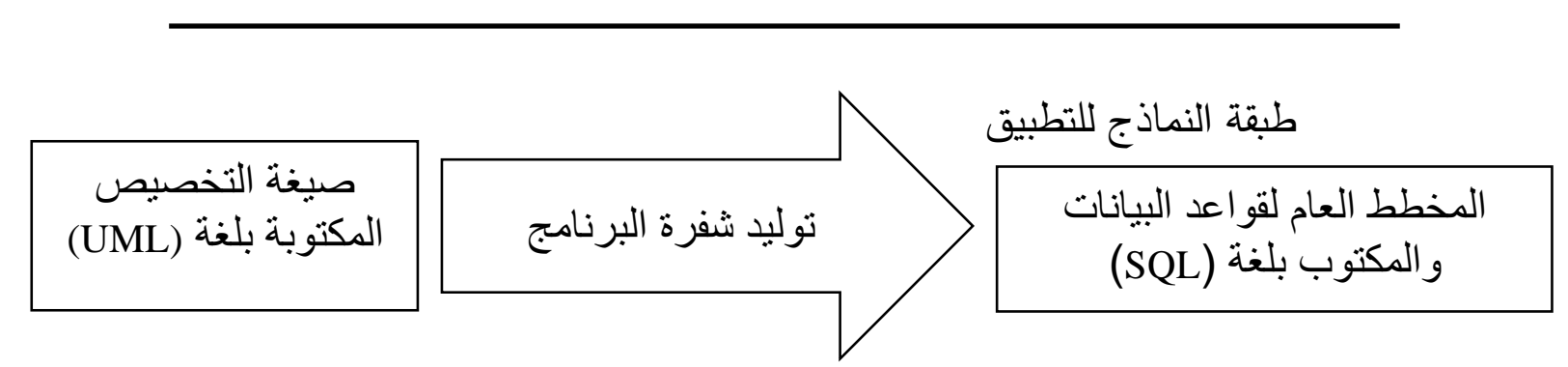

\section{الثكل (2) المعالجة التي تجرى على لغة النماذج الموحدة}

\section{Basic Tools Of Unified Modeling Language}

$$
\text { 3-الأدوات الأساسية للغة النماذج الموحدة. }
$$

تحوي لغـة النماذج الموحدة على أدوات مثل الكيانات، الأصناف، الروابط

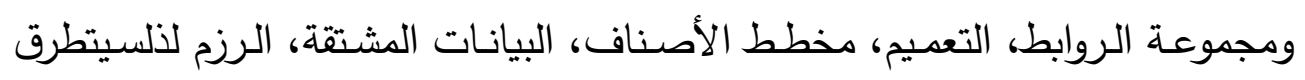

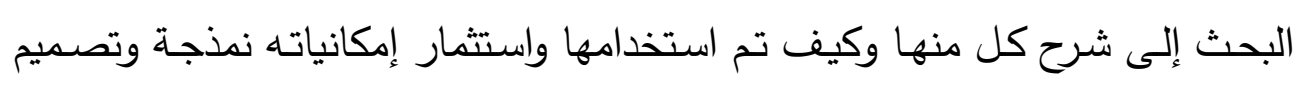

$$
\text { نظام حساب الاندثارات وهي. }
$$

Objects 1-3 الكيانات

تعّد الكيانات الأساس لعمل النموذج ويتم اختيار الكيان من خلال الأسماء

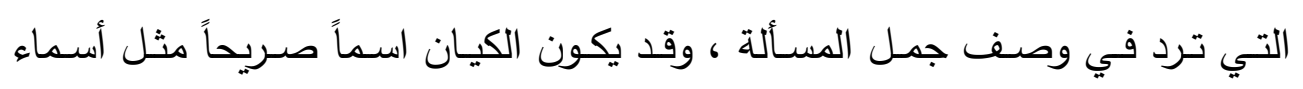
الموظفين (علي، احد...) أو يكون اسماً لأشياء مثل (المادة، ساعات الاشتغال....).

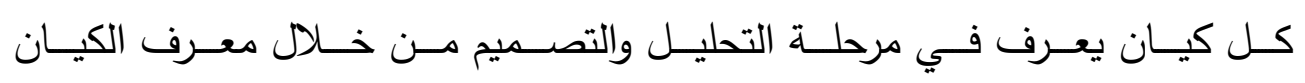
خلان Object Identifier OID

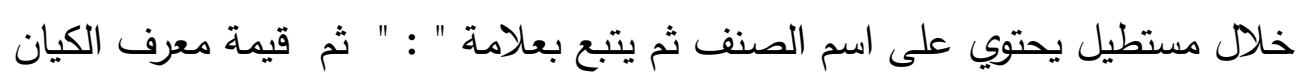

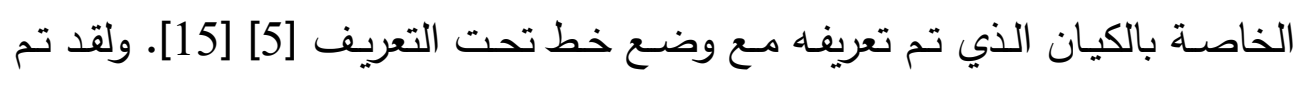
تعريف كيانات متعددة في نظام الاندثارات مثل كيان رقم الحسـاب إذ يمثل الرقم

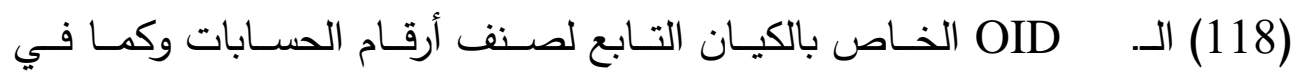

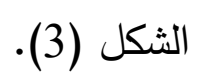




\begin{tabular}{|rr|}
\hline $118:$ & Account_no \\
\hline Accnt_no & $118:$ \\
accnt_nam & $:$ أثاث \\
extin_ratio & : 10 \\
& (3) تعريف الكيان (3)
\end{tabular}

Classes

Attribute هي مجموعـة مـن الكيانـات التي تثـترك بالخصـائص نفسهات

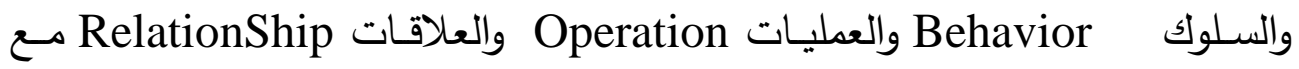
الكيانات الأخرى ويعتمد المدى الخاص بالصنف على التطبيق أو المسألة ـ يمثل الصنف بالرسم من خلال مستطيل يحتوي على:

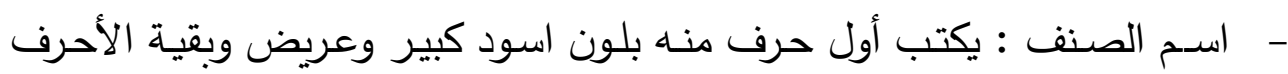
صغيرة ويوضع الاسم في وسط المستطيل .

- خصائص الصنف : تكتب أسماء الخصائص الخاصة بالصنف إلى اليسار .

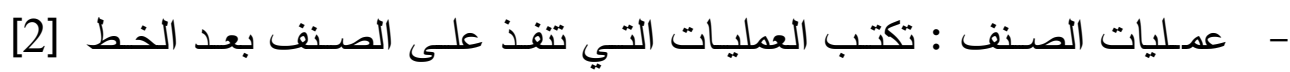

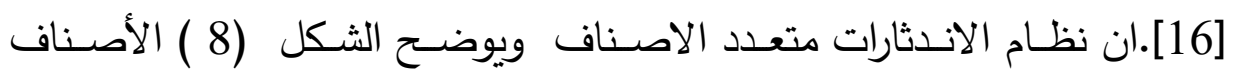

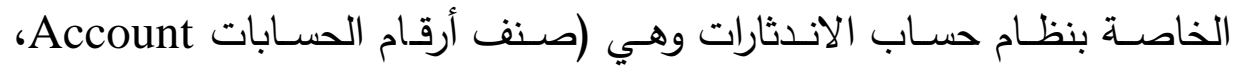

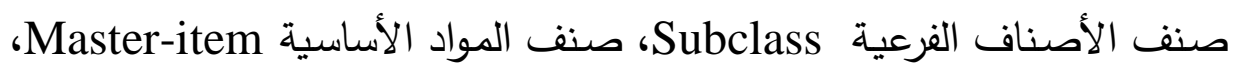

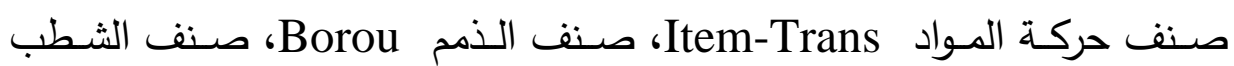
والتحويل Del-Trans ،صنف ساعات الاشتغال Work-hour، صنف الأقسام Department، صنف اللجان Consul، صنف الجهات Agent، صنف أرقام الهواتف Phone-no، صنف طلبات الشراء Order، صنف المواد

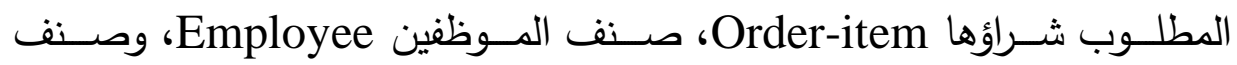

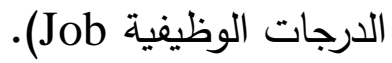


3-3 القيم وخصائص الكيان والخصائص المتعددة

Values, Object Attribute \& Attribute Multiplicity

القيم Values هي جزء من البيانات التي تمثل الأمثلة الخاصـة بالمسألة

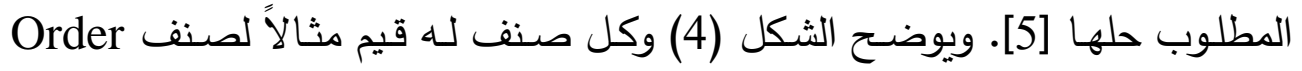
طلبــات الثـــراء ولـــهـ القــيم ‘156324’ , 2000/01/01 , 2000/02/02 , .4000,[65,(10,400,2000/03/01).65]

\begin{tabular}{|l|l|}
\hline \multicolumn{2}{|c|}{ Order } \\
\hline Order_id & $: 156324$ \\
Order_date & $: 2000 / 01 / 01$ \\
Ship_date & $: 2000 / 02 / 02$ \\
Items & $:$ [5 الثكل (4) قيم الصنف \\
total & :4000,2000/03/01),6510] \\
\hline Total valu & $($ )
\end{tabular}

خصائص الكيان Object Attribute هي الصفات التي تعبر عن الصنف[5].

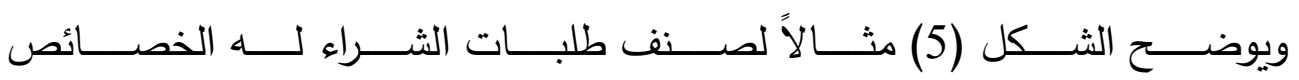
.total , items, ship_date, order_date, id_order

\begin{tabular}{|l|}
\hline \multicolumn{1}{|c|}{ Order } \\
\hline Order_id \\
Order_date \\
ship_date \\
items \\
total \\
\hline total valu ( ) \\
\multicolumn{1}{|l|}{ (5) خصائص الصنف (5) }
\end{tabular}


تعدد الخصـائص Multiplicity Attributes هي احتمـالات القيم التي تأخذها الخصائص تكون إما-قيمة واحدة [1]. - قيماً اختيارية [1,0].

- مجال القيم [الحد الأدنى.. الحد الأعلى] أو [الحد الأدنى ..غير محدد")] [5].

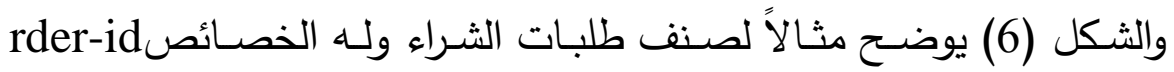
بقيمة [1] , بقيمة [1]] , بقيمة [1tems, [1] بقيمة

[1] بقيمة total , [ *..1]

\begin{tabular}{|ll|}
\hline & Order \\
\hline Order_id & $:[1]$ \\
order_date & $:[1]$ \\
ship_date & $:[1]$ \\
items & $:\left[1 . .^{*}\right]$ \\
total & $:[1]$ \\
\hline \multicolumn{2}{l}{ ( )total valu } \\
\hline
\end{tabular}

الثكل (6) تعدد خصائص الصنف

\section{Class Domain}

3-4 مدى الصنف

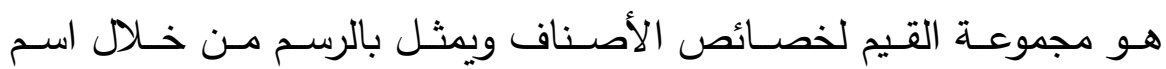
الخاصية ثم ‘:' ويليها نوع الخاصية [5]. وكما في الثكل (8 ) الخـاص بنظسام الاندثارات فصنف ارقام الحسابات Account لله الخصائص accnt-no من نوع

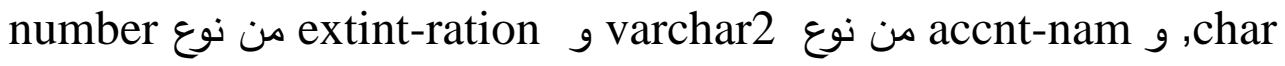

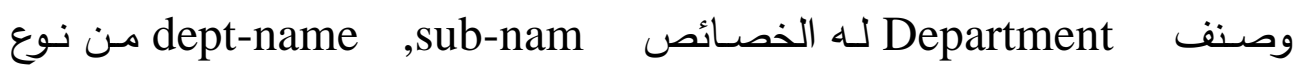
.varchar2

\section{Operation \& Methods}

3-5 العمليات والطرائق

العمليات Operations هي الدوال أو الإجراءات التي يطبقها الكيان الموجود في

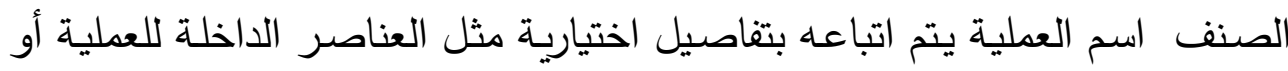


polymorphism النتائج الخارجة منها وبعض العمليات لها صفة تعدد الأشكال [5]. ويوضح ذلك صنف حركة المواد Item-tran في الثكل (8) الذي يحتوي

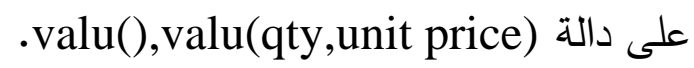
الطرائق Methods تمثل العمليات الخاصة بالصنف والتي يتم تمثلها بالرسم في

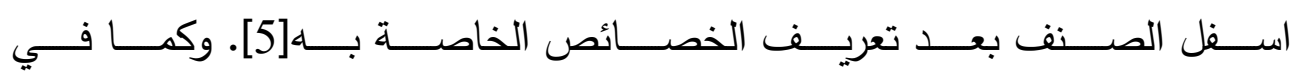
دالة (total valu() والخاصـة بصنف طلبات الثراء Order ودالة () full-name الخاصـة بصنف الموظفين Employee ودالـة item-age الخاصـة بصنف حركـة

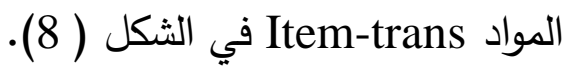

\section{Association \& Multiplicity}

3-6 الروابط المنفردة والمتعددة بين الأصناف

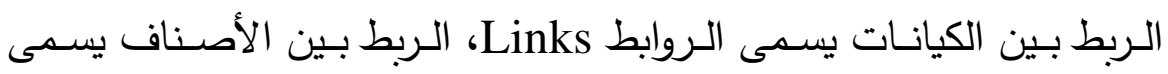

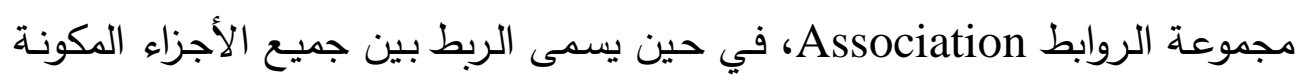

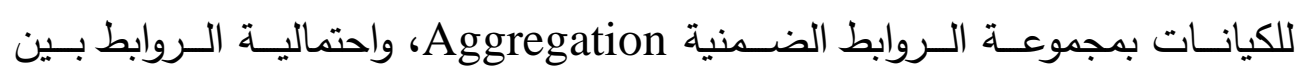
الأصناف تسمى بالتعددية Multiplicity.

-6-3-3- الروابط ومجموعة الروابط ومجموعة الروابط الضمنية

\section{Link \& Association \& Aggregation}

الربط Link وهو الترابط الفيزيائي بين اثثين أو اكثر من الكيانات ويتم تمثيله

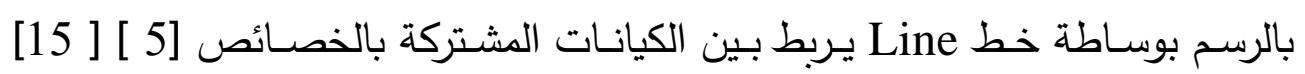

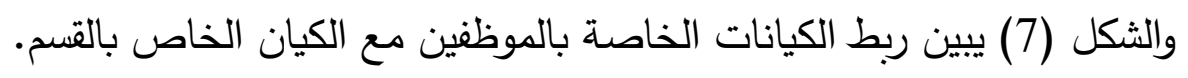




\begin{tabular}{|l|l|}
\hline \multicolumn{2}{|c|}{ Employee : احمد علي } \\
\hline Employee-cod & $125689:$ \\
Emp-nam & احمد علي: \\
\hline
\end{tabular}

\begin{tabular}{|l|r|}
\hline \multicolumn{2}{|c|}{ Employee: محمد 125688} \\
\hline Employee-cod & خالـ \\
Emp-nam & محمود: \\
\hline
\end{tabular}

employee ref

employee ref

\begin{tabular}{|c|c|c|}
\hline 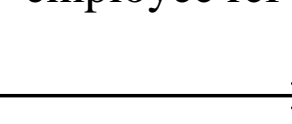 & \multicolumn{2}{|c|}{ الحسابات: Department } \\
\hline & Dept-nam & الحسابات: \\
\hline & Sub-nam & الرواتب: \\
\hline
\end{tabular}

مجموعة الروابط Association هي مجموعة الروابط بين الأصناف التي تشـترك بمجموعـة مـن الخصـائص والهيكليـة المشتركة ويـتم اختيارهـا مـن الأفعـال

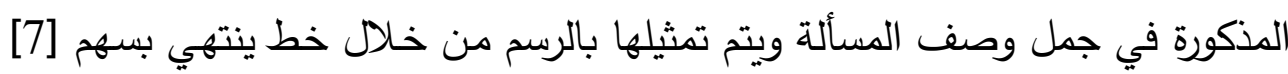

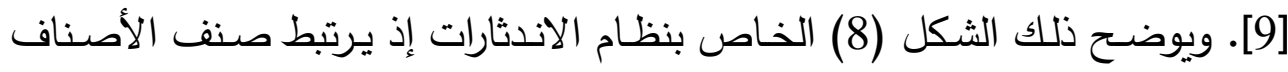

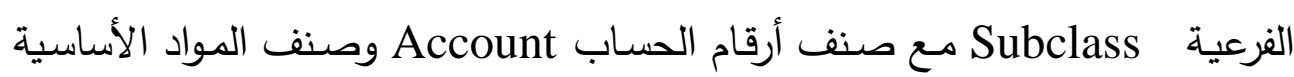

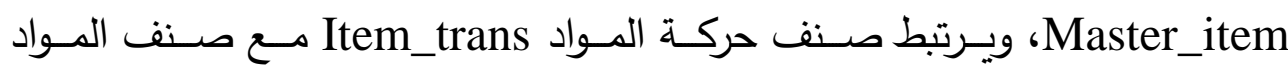
الأساسية Master_item وصنف طلبات الشراء Order، ويرتبط صنف الموظفين مع صنف الأقسام Department وصنف طلبات الشراء Order باستخدام مجموعة الروابط.

مجموعـة الـروابط الضــنية Aggregation هي نـوع مـن الـروابط بـين جميع

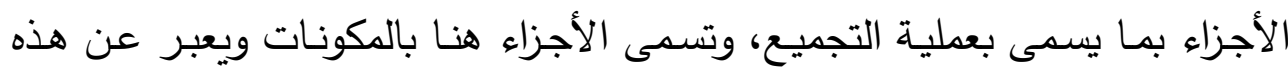

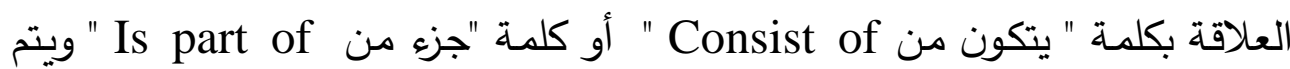
تمثيلها بالرسم على شكل معين مجوف يتجه من الجزء إلى الكل [4]. والثكل (8) 
يوضـح عمليـة الربط بين صـنف طلبات الثـراء Order مـع صـنف مـواد الطلبيـة

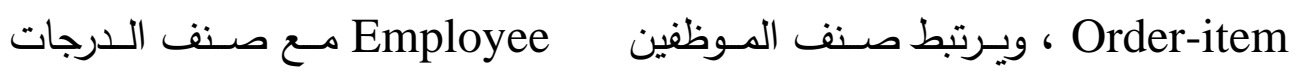
الوظيفية Job ، ويرتبط صنف الجهات Agent مس صع صنف ارقام الهواتف باستخدام مجموعة الروابط الضمنية. Phone_no

\section{Role}

2-6-3

تمثل الوظيفة التي يقوم بها صنف تجاه الآخر ويتم اختيارها من الأسماء التي

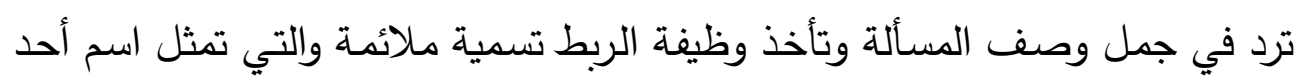
خصائص الكيان للإثـارة إلى مجموعة الروابط وتعّد هذه التسمية خاصية مستعارة pseudo attribute

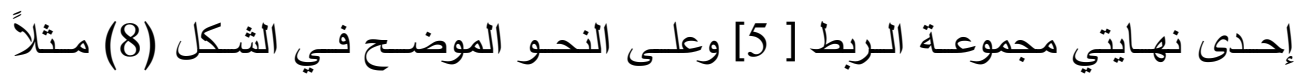
Employee تشير إلى وظيفة العلاقة بين صنف الموظفين Employee-Ref والأقسام Department، وتثير dept_cod إلى وظيفة العلاقة بين صنف الذمم Borou Ref

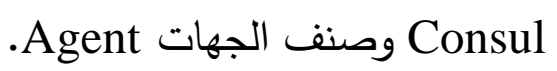

Multiplicity 3-6-3 تعدد الروابط

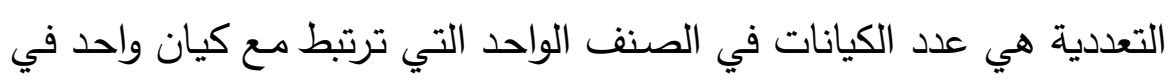

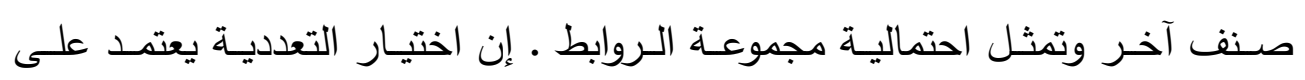

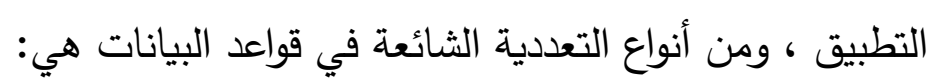

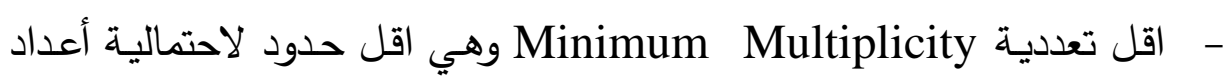
الكيانات المترابطة والقيم المستخدمة هي صفر في حالة عدم توافر احتمالية للربطو واحد في حالة وجوب احتمالية الربط . 
- أعلى تعددية Maximum Multiplicity وهي أعلى حدود لاحتمالية أعداد الكيانات المترابطة والقيم المستخدمة هي واحد أو عدد غير محدد

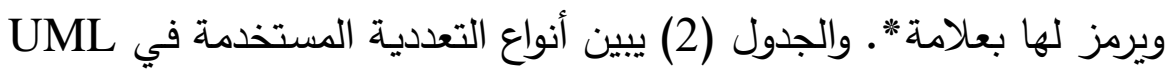
وطريقة تمثيلها بالرسم [6] [9] [16].

\begin{tabular}{|c|c|c|c|}
\hline أعلى تعددية & اقل تعددية & المعنى & رموز لغة النماذج الموحدة \\
\hline واحد & واحد & واحد فقط & ل الصنف \\
\hline غير محدد & صفر & صفر أو اكثر & $* . .0$ \\
\hline واحد & صغر & صفر أو واحد & $1 . .0$ \\
\hline غير محدد & واحد & واحد أو اكثر & 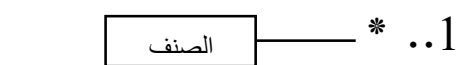 \\
\hline
\end{tabular}

الجدول (2) أنواع التعددية في لغة النماذج الموحدة

\section{Generalization}

التعميم هو العلاقة بين الصنف الرئيس مـع واحـد أو اكثر من الأصناف

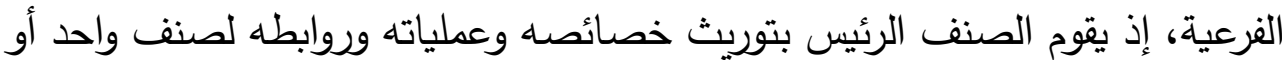

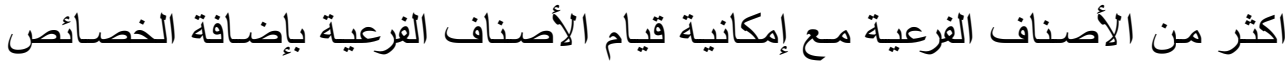

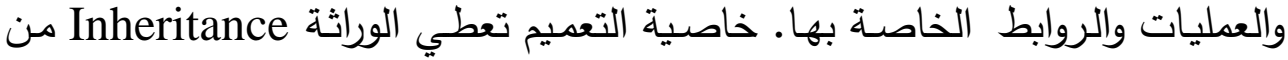
خلال هيكلية العلاقات التي توفر إعادة الاستخدام ويعّد التعديم مهم جداً في بناء الأفكار الخاصــة وتمثيلهـا بـالنموذج إذ يقوم بتتظيم الأصـناف مـن ناحيـة التشــابه والاختلاف وبشكل هرمي إذ يرتبط كل صنف فرعي بصورة مباشرة مع صنف رئيس واحد وهنالك مستويات متعددة من علاقات التعميم .

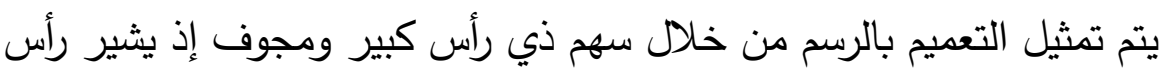

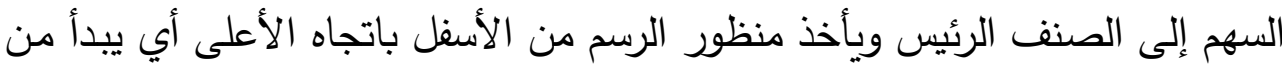

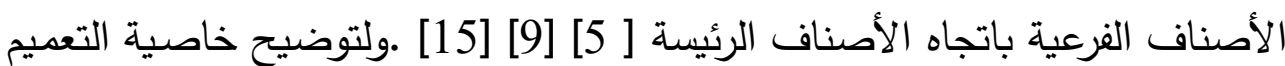


التي يتم على أساسـها الربط بين الأصـناف الرئيسـة والفرعيـة يتم استخدام الميز discriminator

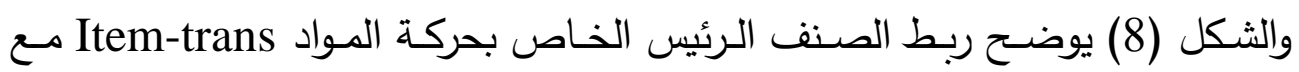

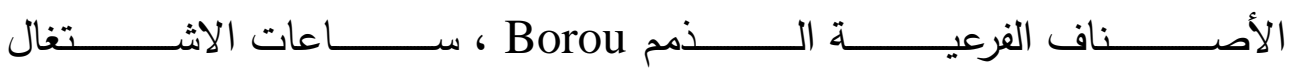
والثـطب Dork-hour

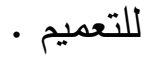

\section{3-3 مخطط الأصناف باستخدام لغة النماذج الموحدة}

\section{UML Class Diagram}

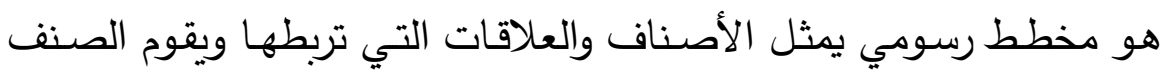
بتمثيل البيانات المجردة [8][14]. والثكل (8) يمثل المخطط العام للأصناف الخاصة بنظام حساب الاندثارات.

Derived Data 3-8 البيانات المشتقة هي البيانـات التي يتم تحديدها واشتقاقها بشكل تـام مـن بيانـات أخرى مثل البـل (الأصناف، الصفات، مجموعة الروابط).لا يتم عرض البيانات المشتقة في التحليل

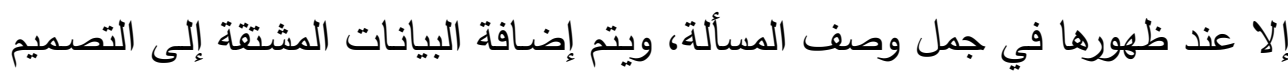
لتبسيط التمثيل وتحسين كفاءته، وفي أثناء التمثيل يتم حساب البيانات المشتقة عند إضله

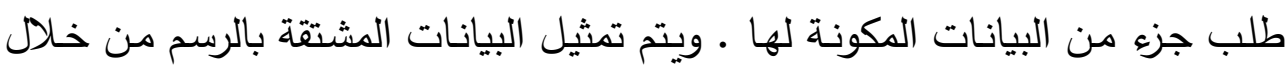

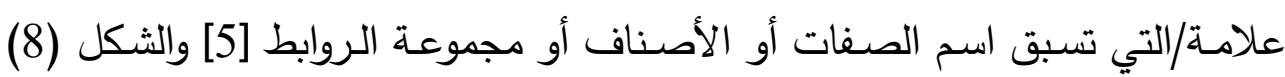
يحتوي على صنف حركة المواد وفيه خاصية عمر المادة التي تمثل بيانات مشتقة.

\section{Packages}

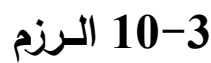

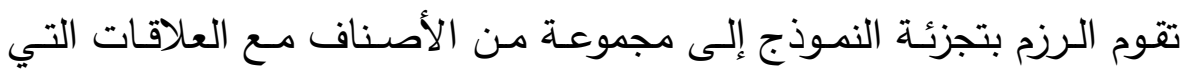
تربطها مما يوفر سهولة الفهم والإدارة والتطوير المستقبلي للنموذج • يتم تمثيل الرزمة التحة 
بالرسم على شكل مستطيل مع إضافة عروةtab إليه وكتابة اسم الرزمة في وسطه.

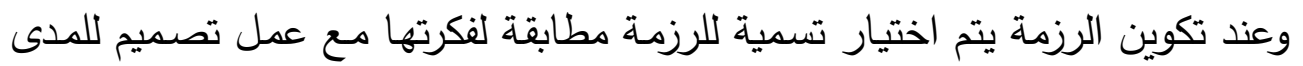

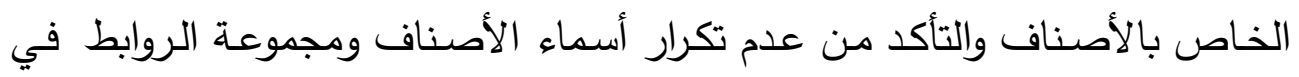

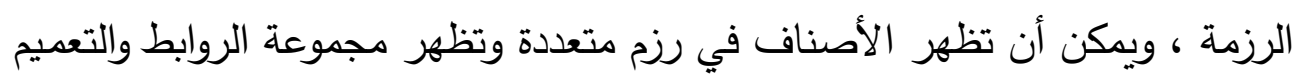

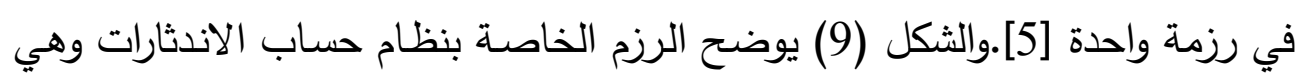

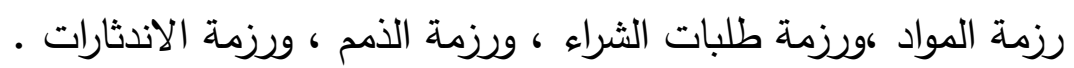

\section{Logical Horizn}

11-3 الأفق المنطقي

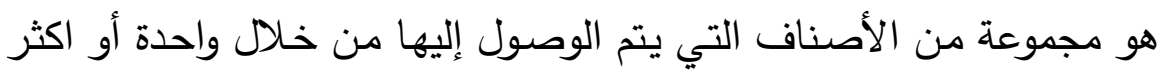

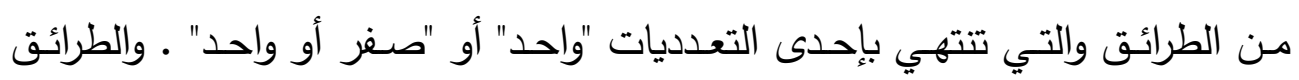

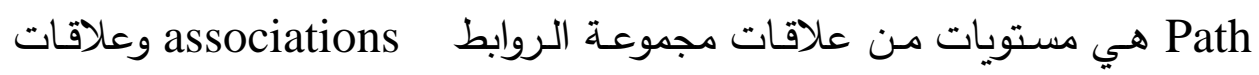

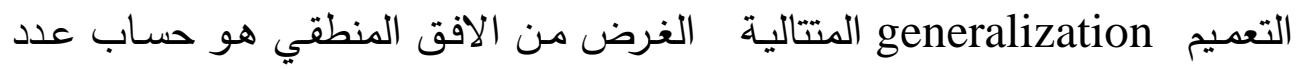
الكيانات والتي تستتتج من الكيان الرئيس ويتم حسابه من خلال رسم المخطط العام

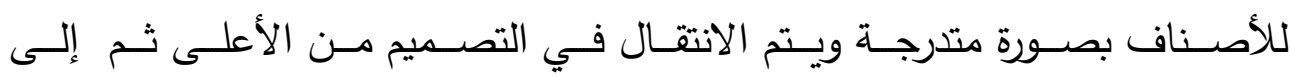
الأسفل [5]. الثكل (10) يبين احتساب الأفق المنطقي لنظسام حسـاب الاندثارات

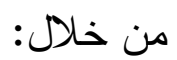

(1) نبدأ بحركة المواد Item-trans)

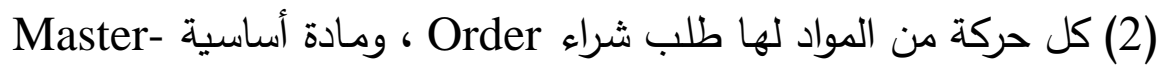

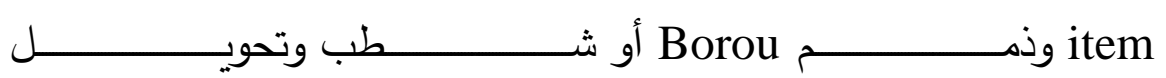
.Work-hour أو ساعات الاشتغال Del-trans (3) ولكل طلب شراء Order لـه مواد مطلوبـة Order-item وموظفون

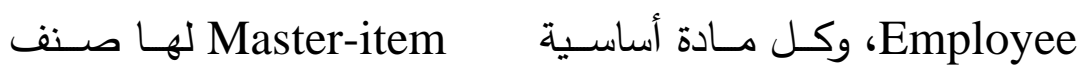

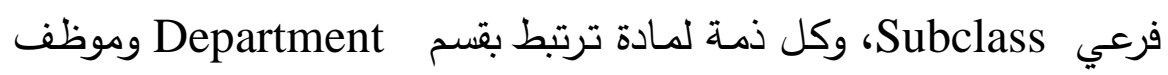


Employee، وكل شطب وتحويل يرتبط مع لجنة Consul وجهة

للتحويل Aggent.

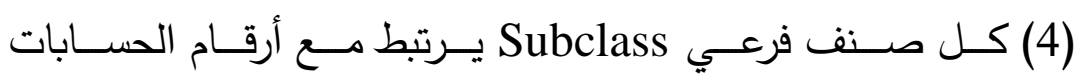

Account وكل موظف Employee يرتبط مع الدرجة الوظيفية

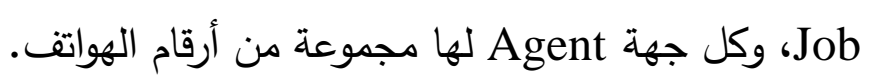

الاستنتاجات

يمكن استخلاص الاستتتاجات التي تم التوصل إليها في البحث على النحو

1- يفضل استخدام النمذجة البيانية الكيانية في تمثيل نموذج قواعد البيانات الكيانية

للانظمة المعقدة ذات الطبيعة التشعبية لكونها توفر :

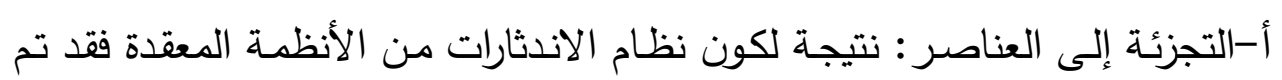

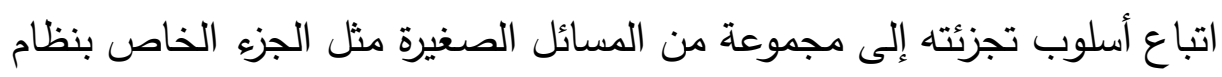

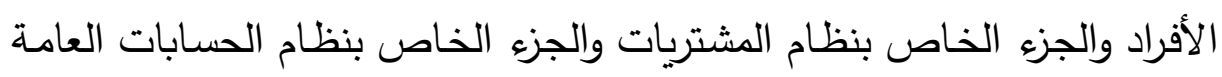

والجزء الخاص بالاندثارات ـ وباستخدام خاصية التجميع تم القيام بتجميع هذه وأهن

الأجزاء بسهولة لتكوين النموذج العام للنظام .

ب-الفهم والإدراك : تم التعامل مـع كل جزء من النماذج السـابقة بوصفه نموذجاً

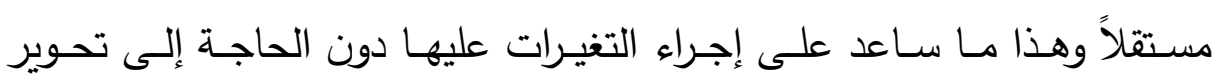

$$
\text { البرنامج بأكمله . }
$$

ج-الحمايـة : إن معالجة الأخطاء التي ظهرئ فرت في مرحلة البرمجة كانت محددة

ضمن كل نموذج دون أن تئثر في بقية النماذج بصورة كاملة .

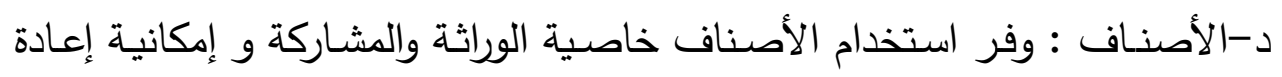

الاستخدام مع تجنب التكرار مقارنة بالنظم العلائقية . 
2- يفضـل استخدام لغــة النهـاذج الموحدة UML لأنهـا تـوفر رمـوزا سـهلة الفهـ والاستخدام ويمكن قراءة النموذج الخاص بالتطبيق من قبل أي شخص لـه معرفة النه بهذه الرموز مما يسهل عملية حفظه واعادة استخدامه من مكتبة البرمجيات والرجوع

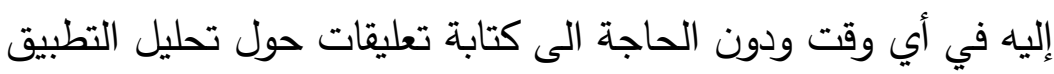

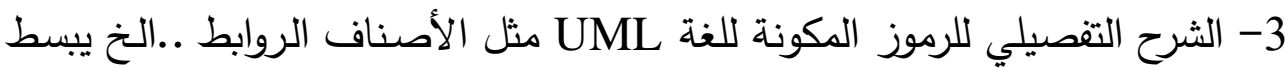
عملية البرمجة ويمكن من تجزئة العمل على فريق عمل من المبرمجين كل واحد منهم يتاول جزءا من المسالة وهذا يؤدي الى تقليل حجم البرنامج وتقليل الوقت

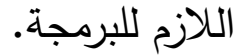



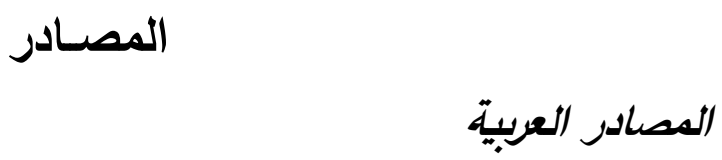

1. الجمهورية العراقية ديوان الرقابة المالية ، 1985. النظام المحاسبي الموحد ، الدار

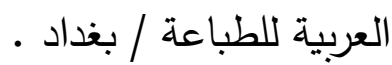

المصادر الأجنبية

2- Johnny O. \& Kasper Q. , 1998 . Object Relational Modeling COT/4-04-V1.0 , http://www.cit.dk /cot/reports/reports/case4/.4-v1,./ COT-4-04-1,.pdf

3- Blaha M. , 1993 . Secondary aspect of Modeling . Journal of Object-Oriented Programming 6,1 (March 1993),15-18.

4- Blaha M. , 1993 . Aggregation of parts of parts of parts . Journal of Object-Oriented Programming 6,5 (September 1993),14-20.

5- Blaha M. , 1998 . Object-oriented Modeling And Design For Database Application , Prentic Hall,Inc.

6- Booch G. ; Jacobson I. ; Rumbaugh J., , 1998 . UML Users Guied . Reading, Massachusetts : Addison-Wesley .

7- Cattell R. , 1991 . Object Data Management : ObjectOriented and Extended Relational Data base System . reading, Massachusetts:Addison-Wesley. .

8- $\quad$;Cutler A. ; Clegg D. , 1997 . Object Database Designeroracle8 Design \& Generation Technology , http://www.techent.oracle.com.

9- Giese H . 2001, .Design Pattern and Software Architecture , http://www.odtug.com/2001 papers / 
sikora.pdf.

10- Feldman F. ; Miller d. , 1996 , Entity Model Clustring : Structring a data model by abstraction . computer journal 29 , 4 (August 1996), 384-360.

11- Jacobson I. ; Booch G. ; Rumbaugh J. , 1998 . UML process book . Reading, Massachusetts : Addison-Wesley .

12- Mauricio J. V. ; Robert Carlson C. , 1995 . MOODD , a method for Object-Oriented Database Design , Data \& Knowledge Engineering 17(1995) 159-181.

13- Mclellan T. , 1995 . Data Modeling : Finding the Perfect Fit , http://www.Data modeling finding the perfect fit.htm

14- Platinum technology , 1998 . Forward Engineering With Oracle 8, http://www.platinum.com.

15- Ratio Group Ltd . 2001 , Persistence : Implementing Object Over a Relation Database version 1.0 , http://www.ratio.co.uk.

16- Rumbaugh J. ; Jacobson I. ; Booch G. , 1998 . UML Refrence Manual book . Reading, Massachusetts : AddisonWesley .

17- Rumbaugh J. ; Jacobson I. ; Booch G. , 1991 . ObjectOriented Modeling \& Design . Englewood Cliffs , New Jersey : prentice Hall . Massachusetts : Addison-Wesley .

18- Woodger Computing Inc , 2001 . Object Data Base , http://www.wci object DB capabilities.htm. 